irreplaceable in the diagnostic approach to lower respiratory tract infections.

By contrast, in our study, no information was collected about viral or mixed infection. Viruses may be isolated in up to $45 \%$ of severe COPD exacerbations, either alone or together with bacteria, and are likely to be present with fever, but their role in sputum purulence is unclear.

In addition, we agree with D. Aggarwal and co-workers about the interest of clarifying the association between sputum purulence and total exposure to antibiotics, but our study was not specifically designed for this purpose. Patients who had previously received antibiotics were excluded because this condition could skew the allocation of cases to each group.

Lastly, is known that purulence is strongly related to colour, although this sign can often be quite subjective. In prospective studies, mucoid sputum is usually described as colourless white, and purulence as ranging from pale yellow to dark green. A recently pooled analysis of clinical trials results in acute exacerbations of chronic bronchitis showed sputum colour to be a stronger predictor of pathogenic microorganism presence than other factors, including sputum purulence [5]. The authors found a significant and consistent relationship between purulence and the presence of a pathogenic bacteria in sputum, although it had less predictive value that the colour of the sample.

We previously evaluated the significant association between patient-reported sputum purulence as an exacerbation criterion and the presence of bacteria in the lower respiratory tract. Sputum was defined as "purulent" when patients reported a change in the colour of spontaneously expectorated samples within the $72 \mathrm{~h}$ prior to admission from uncoloured to yellowgreen. Therefore, the findings of present pilot study support the efficacy and safety of the patient-reported purulence to guide antibiotic treatment.

The change in sputum colour in the course of a COPD exacerbation can be used in self-management plans, leading to early antimicrobial therapy that may reduce the reduction of the course of exacerbation and improve outcomes. In addition, avoiding the use of antimicrobials when the sputum is white will reduce inappropriate antibiotic prescribing and help slow down the appearance of bacterial resistance.
An additional finding increased the confidence in such guidance: nonpurulent sputum was significantly associated with a lower systemic inflammatory response as assessed by serum C-reactive protein concentration. Measurements of plasma biomarkers show the promise to increase the reliability of the assessment of the bacterial aetiology of exacerbation, identifying patients who require antimicrobial treatment.

The findings of our study cohort are the basis to perform a randomised placebo-controlled trial using sputum-guided treatment in nonpurulent COPD exacerbations.

\section{Néstor Soler and Antoni Torres}

Pneumonology Dept, Institute of Thorax, Hospital ClínicIDIBAPS, University of Barcelona, Ciber de Enfermedades Respiratorias, Barcelona, Spain.

Correspondence: A. Torres, Servei de Pneumologia, Institut del Tòrax, Hospital Clínic-IDIBAPS, Villarroel 170, 08036 Barcelona, Spain. E-mail: atorres@clinic.ub.es

Statement of Interest: None declared.

\section{REFERENCES}

1 Soler N, Esperatti M, Ewig S, et al. Sputum purulence-guided antibiotic use in hospitalised patients with exacerbations of COPD. Eur Respir J 2012; 40: 1344-1353.

2 Anthonisen NR, Manfreda J, Warren CP, et al. Antibiotic therapy in exacerbations of chronic obstructive pulmonary disease. Ann Intern Med 1987; 106: 196-204.

3 Stockley RA, ÒBrien C, Pye A, et al. Relationship of sputum colour to nature and outpatient management of acute exacerbations of COPD. Chest 2000; 117: 1638-1645.

4 Soler N, Agusti C, Angrill J, et al. Bronchoscopic validation of the significance of sputum purulence in severe exacerbations of chronic obstructive pulmonary disease. Thorax 2007; 62: 29-35.

5 Miravitlles M, Kruesmann F, Haverstock D, et al. Sputum colour and bacteria in chronic bronchitis exacerbations: a pooled analysis. Eur Respir J 2012; 39: 1354-1360.

\title{
Who and what should we rely on in early diagnosis of idiopathic pulmonary fibrosis?
}

\section{To the Editor:}

In a recent European Respiratory Journal editorial, COTTIN and CORDIER [1] ranked velcro crackles among diagnostic clues in early idiopathic pulmonary fibrosis (IPF). We agree that auscultation is essential for early detection of IPF, but physical examinations should be integrated into a more comprehensive diagnostic strategy. This raises a question: who identifies IPF patients, and by what means?
IPF is a cryptogenic form of usual interstitial pneumonia (UIP), which is best confirmed pathologically or, to a lesser extent, by a radiographic UIP pattern [2]. However, the low incidence (two to 29 cases per 100,000$)$ makes it cost-ineffective to screen for IPF/UIP by computed tomography (CT) in general populations, let alone by lung biopsy [1,2]. Alternatively, clinical features are more feasible and suggestive of IPF as well. The aggregate of three or more out of four characteristics (age $>50 \mathrm{yrs}$, a gradual 
onset of dyspnoea, a duration of illness of $\geqslant 3$ months and the presence of bibasilar velcro crackles) indicates, and was formally designated as, the minor diagnostic criteria for IPF [2]. Based on the presence of inspiratory fine crackles or a history of occult-onset dyspnoea that progresses gradually, every physician is able to list IPF among the candidate diseases and resort to CT scan or lung biopsy to verify this [3]. Velcro-like rales do not establish the diagnosis of IPF exclusively, but rather warrant further investigations. A past medical or environmental history is thus more helpful for narrowing down the suspects. For example, a history of recent radiotherapy or chronic asbestos exposure illuminates the diagnosis of radiation pneumonitis or asbestosis, respectively. A primary diagnosis of IPF is more confidently made by combining physical examination findings and medical histories together than by each one separately.

Though it is supposed that the majority of IPF patients are identified by physicians [1], radiologists also contribute a lot. Computer-aided analysis recognises early interstitial lung diseases with a diagnostic sensitivity of $80.0 \%$ and specificity of $85.7 \%$ in low-dose CT images [4]. More recently, in an Italian lung cancer screening project, two $(0.3 \%)$ typical UIP patterns out of 692 heavy smokers were distinguished via low-dose, thin-section CT [5]. For the foreseeable future, radiologists will probably identify an increasing number of IPF patients by lowdose CT scan in lung cancer screening programmes.

In conclusion, the importance of velcro crackles in diagnosing early IPF increases when analysed together with medical histories, or even with CT images if available. Both physicians and radiologists are ready to make an initial diagnosis of IPF, which is confirmed by lung biopsy in qualified facilities. However, Dr Laennec would be glad to see stethoscope, his most renowned invention, being used by modern doctors as a flashlight beaming on the road towards early IPF.

Xiaoqian Chen*, Xiaoju Zhang", Xiu Li* and Zheng Wang* *Dept of Respiratory and Critical Care Medicine, Hefei No. 1 People's Hospital, Hefei, and "Dept of Respiratory and Critical Care Medicine, Municipal Hospital of Henan Providence, Zhengzhou, China.

Correspondence: Z. Wang, Dept of Respiratory and Critical Care Medicine, Hefei No. 1 People's Hospital, Huaihe Road 390\#, Hefei, Anhui, China. E-mail: santawang99@163.com

Statement of Interest: None declared.

\section{REFERENCES}

1 Cottin V, Cordier J. Velcro crackles: the key for early diagnosis of idiopathic pulmonary fibrosis? Eur Respir J 2012; 40: 519-521.

$2 \mathrm{du}$ Bois RM. An earlier and more confident diagnosis of idiopathic pulmonary fibrosis. Eur Respir Rev 2012; 21: 141-146.

3 Fell CD, Martinez FJ, Liu LX, et al. Clinical predictors of a diagnosis of idiopathic pulmonary fibrosis. Am J Respir Crit Care Med 2010; 181: 832-837.
4 Park SC, Tan J, Wang X, et al. Computer-aided detection of early interstitial lung diseases using low-dose CT images. Phys Med Biol 2011; 56: 1139-1153.

5 Sverzellati N, Guerci L, Randi G, et al. Interstitial lung diseases in a lung cancer screening trial. Eur Respir J 2011; 38: 392-400.

DOI: $10.1183 / 09031936.00142012$

\section{From the authors:}

C. Xiaoqian and colleagues comment on our recent editorial [1], in which we emphasised the potential role of "Velcro" crackles at auscultation for earlier diagnosis of idiopathic pulmonary fibrosis (IPF). They are right to underline that "velcro" crackles were formerly one of the minor criteria for the diagnosis of IPF in the absence of a lung biopsy, and to discuss the role of highresolution computed tomography (HRCT) of the chest.

Although "velcro" crackles alone are undoubtedly not specific of IPF, their finding should prompt the clinician to perform further investigations including chest HRCT, contributing to the early diagnosis of IPF, whatever the symptoms or the context that initially motivated lung auscultation. We further consider that pulmonary auscultation should still be included in the initial steps of the diagnostic algorithm in patients with chronic dyspnoea, especially in those with progressive dyspnoea, as well as in patients with chronic dry cough.

The contribution of HRCT in diagnosing IPF is well established, especially in light of the recent international guidelines, which state that in patients without identifiable cause of interstitial lung disease, a HRCT pattern of usual interstitial pneumonia (UIP) with honeycombing is diagnostic of IPF, obviating the need of a lung biopsy [2]. However, such patients, in whom the definite diagnosis of IPF can be made without a lung biopsy, already have a well-established and irreversible disease. Honeycombing on imaging unfortunately reflects our current failure to detect IPF at an earlier stage, with a risk for patients with markedly altered lung function tests of being excluded from clinical trials and even of being denied pirfenidone therapy. Therefore, we strongly advocate that patients with clinically suspected IPF and a pattern of possible UIP at imaging be given the chance of an early diagnosis confirmed by lung biopsy, even in the case of only mild symptoms and preserved lung function, if the benefit/risk ratio of a biopsy is favourable. Observing the development of honeycombing at imaging and the decline in lung function tests in a given patient long known to have crackles at auscultation and interstitial changes at imaging merely demonstrates that diagnostic (and possibly therapeutic) decisions are long overdue in this patient.

Improving the ability of community physicians to acknowledge "velcro" crackles at lung auscultation and especially to initiate appropriate investigations may be the only key for the earlier diagnosis of IPF: let us have the chance to hear crackles (and to diagnose and possibly treat IPF) before we see honeycombing.

\section{Vincent Cottin and Jean-François Cordier}

Reference Centre for Rare Pulmonary Diseases, Hospices Civils de Lyon, Louis Pradel Hospital, Claude Bernard University Lyon 1, Lyon, France. 